P60 (continued)

or practice-base evidence. Following review for readability, the e-scan was pilot tested in 15 pantry sites.

Conclusions and Implications: The e-scan represents current best practices within food assistance settings. A revised e-scan will be field tested, and a corresponding resource guide will be developed to help pantries improve their assessment scores.

Funding: USDA

\section{P61 Lessons Learned About Rural Food Retail From a Community Level Obesity Prevention Grant}

Janie Burney,PhD, RD, jburney@utk.edu, University of Tennessee Extension, 2621 Morgan Circle, Knoxville, TN 37996; Cori Sweet, BS, University of Tennessee Extension; Karen Franck, PhD; Tennille Short; Rachel Erwin

Objective: To develop and implement strategies designed to increase healthy food options in small retail food outlets. Theory, Prior Research, Rationale: This project is guided by Community-Based Participatory Research model where community members are actively involved in deciding the focus and the implementation of the intervention. Small food retail environments were chosen because they were identified as important contributors to healthy eating patterns among community residents.

Description: A comprehensive needs assessment was conducted through 13 focus groups (122 participants) and 438 surveys. Eleven retail stores were assessed using the Nutrition Environment Measures Survey (NEMS). From the needs assessments, a county-wide social marketing campaign called, BE MORE, was implemented consisting of positive messages and images designed to encourage healthful eating. Campaign materials were developed for retail stores. Evaluation: During the spring of 2017, telephone surveys with community members will be conducted to assess awareness and impact of BE MORE and NEMS assessments will be repeated.

Conclusions and Implications: Conclusions thus far are related to the process of engaging stores. Smaller, independent stores were more likely to participate compared to larger, chain stores. Signage for windows, floors and walls were highly desirable. Smaller signs, banners on stands, window clings and point-of-decision prompts were most popular while large signs and floor clings were less useful.

Funding: Center for Disease Control and Prevention

\section{P62 University Websites Indicate a Lack of Policy Support for Disease Prevention and a Healthy Environment}

Gillian Kelly, Syracuse University; Marlei Simon, BS, RDN; Janette Bedoyan; Abigail Case; Wing Cheung; Natasha Jackson; Sarah Colby, PhD, RD, University of Tennessee; Tanya Horacek, PhD, RD, thoracek@syr.edu, Syracuse University, 558 White Hall, Syracuse, NY 13244
Objective: To describe the status of wellness policies for a sample of university campuses. Wellness policies may create sustainable programs and environments to encourage healthy behaviors.

Study Design, Setting, Participants: The Policy, Opportunities, Initiatives, Notable Topics Survey (POINTS) Audit evaluates university websites for 34 wellness policy topics organized into four sub-scales: stimulants (smoking/alcohol/drugs); disease prevention (DP) (education/ screening), active environment (AE) (closed-campus, sustainable transportation), and nutritious sustainable food systems (NSFS) (food taxes, labels). A 3-point scale was created to assess stage of policy development $(0=$ no policy; $1=$ initiative/interventions; $2=$ written policy). Research assistants were trained to use the audit to conduct comprehensive website reviews (interrater reliability $\alpha=.82)$. Fifty distinct University websites were reviewed (variable sizes, from 30 different states, 50\% private).

Outcome Measures and Analysis: Computed policy strength sub-scores $=$ ((policies + initiatives $) /$ total maximum policy sub-scale points) $[0,100]$. Total score is the average of the four sub-scores. Non-parametric tests and logistic regression were used to compare sub-scores and total score.

Results: Universities have few wellness policies posted on their websites, with moderate mean total score of $57.7 \pm 6.9$ and mean sub-scores: DP $45.3 \pm 11.3 ; \mathrm{AE}$ $45.3 \pm 21.2$; and NSFS 49.2 \pm 6.5 . Most campuses had strong policy support regarding stimulants $(97.5 \pm 9.1)$; Total policy scores were most predicted by $\mathrm{AE}(\beta=.76)$, and DP $(\beta=.407)(\mathrm{p}<0.001)$. Small schools $(<7500 \mathrm{stu}-$ dents) had significantly lower DP scores than medium/ large $(>15,000)$. A few interesting policies relating to fundraising and health-related course requirements will be described.

Conclusions and Implications: An opportunity exists to advocate for policy support beyond stimulant use. Information provided on university websites may not accurately reflect existence of wellness policies. Further research needs to be conducted to validate this tool.

Funding: USDA, Syracuse University

\section{P63 Accuracy of Parent-Reported Feeding Practices and Implications for Behavioral Interventions}

Lisa Fries, PhD, lisa.fries@rdls.nestle.com, Nestlé Research Center Case, Postale 44, Lausanne, VD Switzerland 1000; Nathalie Martin, PhD, Nestlé Research Center; Klazine van der Horst, PhD

Objective: Feeding practices research has largely been based on parents' self-report, but it is unclear whether this reflects actual feeding practices. We aimed to explore which feeding practices were more accurately reported by parents through comparison with behavioral observation.

Study Design, Setting, Participants: Sixty American parents with toddlers video-recorded their child's dinner

Continued on page $S 48$ 
P63 (continued)

at home and completed questionnaires about their feeding practices. The video was coded for parental feeding practices.

Outcome Measures and Analysis: Questionnaire responses of "never," "rarely" were considered correct if the behavior was not observed in the video; responses of "most of the time," "always" were correct if the behavior was observed at least once during dinner. "Sometimes" responses were excluded from analysis. Behaviors that corresponded $>70 \%$ of the time were considered to be reported accurately.

Results: Parents accurately reported the following behaviors: allowing child to eat as much as he wants, helping child to eat, using neutral prompts to eat, having television/screen on during meal, offering non-food rewards for eating, and hurrying child to eat faster. Parents' responses were not accurate for: using reasoning to convince child to eat, allowing child to choose foods, asking child to clean his plate, and offering food rewards for eating.

Conclusions and Implications: Possible explanations why some behaviors are more accurately reported will be discussed. Behaviors which parents can report accurately can be addressed directly through interventions because parents are already aware of using these mealtime behaviors. For less accurately reported behaviors, additional awareness may need to be created through education before parents will be able to recognize and change their behaviors. Funding: Nestle Nutrition

\section{P64 An Exploratory Look at the Role of Childcare Providers as a Support and Resource for Breastfeeding Mothers}

Alexandra Lundquist, BS, lundqui2@illinois.edu, University of Illinois at Urbana-Champaign, 1105 West Nevada, Urbana, IL 61801; Brent McBride, PhD, University of Illinois at Urbana-Champaign;

Sharon M. Donovan, PhD, RD; Chigozirim Asonye;

Alexandra Kieffer; Aubrey Toosley

Objective: Research has examined workplace, healthcare, and family factors in support for the breastfeeding mother, whereas few studies have considered the available support in childcare settings. Breastfeeding continuation in the childcare setting involves the co-commitment and active cooperation between mother and provider. We hypothesized that the transition to non-parental care represents a vulnerable change for first-time mothers where increased support may be needed to facilitate the continuation of breastfeeding, and that mothers overlook childcare as a potential source of influence and support.

Study Design, Setting, Participants, Intervention: Utilizing Bronfenbrenner's Ecological Model as a theoretical framework for the development and evolution of the breastfeeding experience, a semi-structured interview was developed to guide data collection. First-time mothers $(n=25)$ were recruited for interviews.
Outcome Measures and Analysis: Concepts and themes that comprise mothers' experiences with, and perceptions of, navigating the transition to childcare while breastfeeding were identified using Thematic Analysis.

Results: Initial findings suggest that upon the transition to non-parental care, there is a shift in the breastfeeding relationship from one with the infant to one with the breast pump that mothers find less rewarding. Further, mothers do not critically evaluate childcare providers' support for breastfeeding, having only superficial conversations with providers regarding breastfeeding.

Conclusions and Implications: These results demonstrate the need for education that empowers mothers to find support in their childcare provider and provides resources for improving the breastfeeding knowledge of providers. Findings also underscore the need to recognize childcare as a viable source of support for the breastfeeding mother.

Funding: Illinois Transdisciplinary Obesity Prevention Program, National Dairy Council

\section{P65 Are Children Who Attend Family Child- Care Homes Meeting Dietary Recommendations at Home?}

Noereem Mena, MS, LDN, RD, mena23@uri.edu, University of Rhode Island, 125 Fogarty Hall, 41 Lower College Roadd, Kingston, RI 02881; Maggie Tsai, BS, University of Rhode Island; Patricia Risica, DrPH, Brown University; Kim M. Gans, $P h D$, University of Connecticut; Ingrid Lofgren, $P h D, M P H, R D$, University of Rhode Island; Kathleen Gorman, PhD; Alison Tovar, PhD, MPH

Objective: To describe food consumption of children (2-5 years) who attend family child-care homes $(\mathrm{FCCH})$ at home, relative to dietary guidelines.

Study Design, Setting, Participants: Parents of children in FCCHs currently enrolled in a cluster randomized intervention were recruited to participate in an ongoing sub-study to capture foods and beverages their children consume at home.

Outcome Measures and Analysis: Parents $(n=18)$ reported the foods/beverages their children consumed from pickup time to bedtime via two 24-hour recalls. One-sample $\mathrm{t}$ tests and Wilcoxon Signed-rank test, with Bonferroni corrections, were run to test for differences between food groups consumed outside of the FCCH compared to dietary guidelines, assuming that one-third to one-half of total daily nutrient intake should be consumed away from the FCCH. Outcome variables included total energy, refined and whole grains, fruit, and vegetable intake.

Results: Foods consumed outside of child-care are exceeding recommendations for total energy intake, added sugar and refined grains $(\mathrm{p}<0.0001)$. Intake of whole grains and fruit also exceeded recommendations $(\mathrm{p}<0.0001)$, however, vegetable intake fell below both the one-third and one-half proportion recommendations for consumption outside of child-care, mean intake of 0.27 cups vs. recommended intake of 0.3-0.5 cups, $\mathrm{p}<0.0001$.

Conclusion and Implications: Excess energy intake is occurring outside of the FCCH. Yet, children are still not

Continued on page $S 49$ 